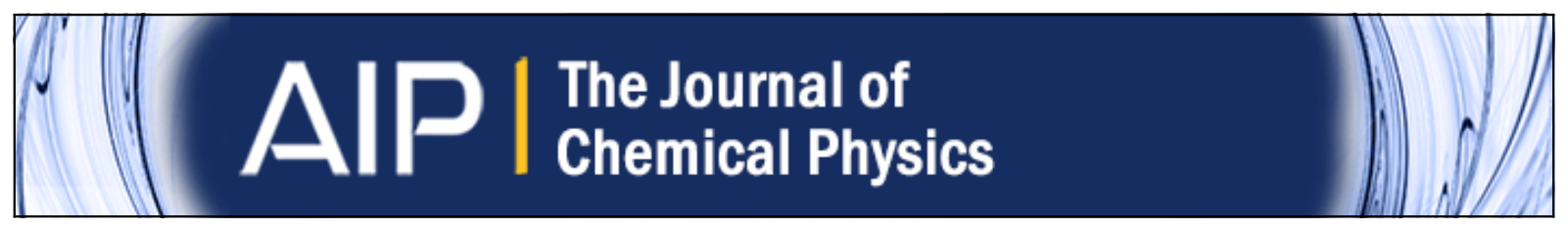

X-ray absorption from large molecules at metal surfaces: Theoretical and experimental results for Co-OEP on $\mathrm{Ni}(100)$

C. S. Guo, L. Sun, K. Hermann, C. F. Hermanns, M. Bernien, and W. Kuch

Citation: The Journal of Chemical Physics 137, 194703 (2012); doi: 10.1063/1.4765373

View online: http://dx.doi.org/10.1063/1.4765373

View Table of Contents: http://scitation.aip.org/content/aip/journal/jcp/137/19?ver=pdfcov

Published by the AIP Publishing

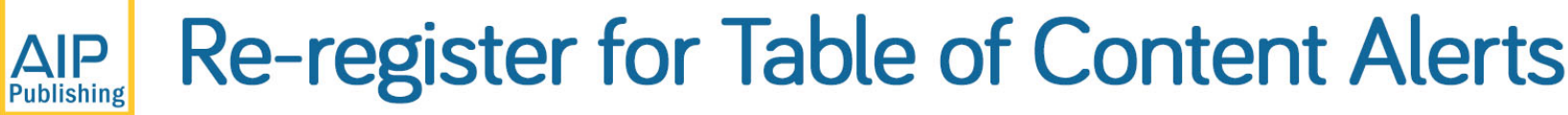

\section{Create a profile.}




\title{
X-ray absorption from large molecules at metal surfaces: Theoretical and experimental results for Co-OEP on $\mathrm{Ni}(100)$
}

\author{
C. S. Guo, ${ }^{1}$ L. Sun, ${ }^{1,2}$ K. Hermann, ${ }^{1}$ C. F. Hermanns, ${ }^{2}$ M. Bernien, ${ }^{2}$ and W. Kuch ${ }^{2}$ \\ ${ }^{1}$ Inorganic Chemistry Department, Fritz-Haber-Institut der MPG, Faradayweg 4-6, D-14195 Berlin, Germany \\ ${ }^{2}$ Institute for Experimental Physics, Freie Universität Berlin, Arnimallee 14, D-14195 Berlin, Germany
}

(Received 29 June 2012; accepted 18 October 2012; published online 21 November 2012)

\begin{abstract}
Metal octaethylporphyrins (M-OEP), $\mathrm{M}-\mathrm{N}_{4} \mathrm{C}_{20} \mathrm{H}_{4}\left(\mathrm{C}_{2} \mathrm{H}_{5}\right)_{8}$, adsorbed at a metallic substrate are promising candidates to provide spin dependent electric transport. Despite these systems having been studied extensively by experiment, details of the adsorbate geometry and surface binding are still unclear. We have carried out density functional theory calculations for cobalt octaethyl porphyrin (Co-OEP) adsorbate at clean and oxygen-covered $\mathrm{Ni}(100)$ surfaces as well as for the free Co-OEP molecule where equilibrium structures were obtained by corresponding energy optimizations. These geometries were then used in calculations of Co-OEP carbon and nitrogen 1s core excitations yielding theoretical excitation spectra to be compared with corresponding K-edge x-ray absorption fine structure (NEXAFS) measurements. The experimental NEXAFS spectra near the carbon K-edge of Co-OEP bulk material show large intensity close to the ionization threshold and a triple-peak structure at lower energies, which can be reproduced by the calculations on free Co-OEP. The experimental nitrogen K-edge spectra of adsorbed Co-OEP layers exhibit always a double-peak structure below ionization threshold, independent of the layer thickness. The peaks are shifted slightly and their separation varies with adsorbate-substrate distance. This can be explained by hybridization of $\mathrm{N} 2 \mathrm{p}$ with corresponding $3 \mathrm{~d}$ contributions of the Ni substrate in the excited final state orbitals as a result of adsorbate-substrate binding via N-Ni bond formation. () 2012 American Institute of Physics. [http://dx.doi.org/10.1063/1.4765373]
\end{abstract}

\section{INTRODUCTION}

Owing to its elemental selectivity, the effect of x-ray magnetic circular dichroism (XMCD) provides the unique capability for studying the magnetization of paramagnetic molecules in contact with a ferromagnetic substrate. Magnetic metalorganic molecules, stabilized at solid substrates, are considered the main building blocks of future molecular spintronic devices. Here the adsorbate-substrate interaction in general has a strong influence on the electronic, structural, and magnetic properties of adsorbed molecules. Studying the influence of a well-defined substrate on the properties of magnetic metalorganic molecules is thus of high importance.

Porphyrin molecules are of particular interest, since their planar four-fold coordinated structure allows the incorporation of metal ions in the center which can be magnetic and used to perform binary logic operations. For example, attaching a photo-switchable ligand to a Ni porphyrin molecule in solution allows to reversibly switch the spin state of the molecules by visible light. ${ }^{1}$ On surfaces, coupling between the magnetic moment of the molecule and that of a metallic ferromagnetic substrate has been observed for several metalloporphyrins. ${ }^{2-5}$ Inserting oxygen atoms between the porphyrin molecules and the substrate can switch the molecule-substrate coupling to being antiferromagnetic. ${ }^{6,7}$ Further, co-adsorption and thermal desorption of $\mathrm{NO}$ at the empty ligand site of adsorbed porphyrin molecules was found to allow reversible manipulation of the molecular spin state ${ }^{8}$ or the magnetic coupling with the ferromagnetic substrate layer. ${ }^{9}$

While $\mathrm{x}$-ray absorption spectra near the L-2,3 edge of the metal center of metalloporphyrins reveal oxidation and spin states, polarization-resolved absorption spectra at the nitrogen and carbon K-edges are essential for a complete understanding of the molecule-substrate interaction. The latter contain valuable information on the molecular electronic state, ${ }^{10}$ its orientation, ${ }^{3,11-13}$ and substrate-induced deformation. ${ }^{14}$ In the case of iron porphyrins on metallic substrates the four nitrogen atoms of the porphyrin ring are held responsible for the ferromagnetic coupling of the molecules with the substrate where they mediate a $90^{\circ}$ superexchange coupling between the $\mathrm{Fe}$ ion and the substrate atoms. ${ }^{3}$ In contrast, on the metal substrate covered by half a monolayer of atomic oxygen the nitrogen atoms do not significantly contribute to this coupling, which then is of the $180^{\circ}$ superexchange type. These magnetic couplings between metal porphyrin and metal substrate seem to be of more general importance and may also apply to other porphyrin adsorbates, such as cobalt octaethyl porphyrin (Co-OEP) or Mn-OEP, which, so far, have not been studied in detail. Other examples include Mn-TTP adsorbed at a Co substrate. 5,7

In this work, we present detailed theoretical results of $\mathrm{C}$ and $\mathrm{N} \mathrm{K}$-edge $\mathrm{x}$-ray absorption spectra obtained by density functional theory (DFT) for free Co-OEP molecules as well as for adsorbed Co-OEP at clean and oxygen-covered single crystal $\mathrm{Ni}(100)$ substrate using cluster models. The results 
are interpreted by electronic structure analyses of excited final states and compared with measured (polarization-resolved and integrated) K-edge x-ray absorption fine structure (NEXAFS) spectra for corresponding adsorbate systems and a Co-OEP bulk reference sample.

In Sec. II we introduce the models and discuss details of the computational methods used in the spectrum calculations. Section III describes experimental details connected with sample preparation and NEXAFS measurements while Sec. IV presents results and discussion. Finally, Sec. V summarizes our conclusions.

\section{THEORETICAL DETAILS}

\section{A. Geometric and electronic structure, cluster models}

The free Co-OEP molecule is composed of an almost planar central $\mathrm{CoN}_{4} \mathrm{C}_{20} \mathrm{H}_{4}$ part which is surrounded by eight ethyl groups pointing to the same side away from the plane, yielding a $\mathrm{CoN}_{4} \mathrm{C}_{36} \mathrm{H}_{44}$ molecule, see Fig. 1. Due to its fourfold rotational symmetry there is only one type of nitrogen while five different carbon species, labeled C1 to C5 appear in Co-OEP, as shown in the figure.

The Co-OEP molecule adsorbed at metal substrate was reported to stabilize with its plane parallel to the surface. ${ }^{13,15}$ This adsorbate geometry is confirmed by theoretical optimizations for Co-OEP on Ni (100). Here the substrate surface is modeled by one fixed Ni layer of (100) orientation (forming a square lattice) while the Co-OEP adsorbate is relaxed at three different lateral surface sites: With the central Co atom at the four-fold hollow surface site, on top of a Ni center, and at a two-fold bridging surface site. For all sites the equilibrium geometry is obtained by periodic DFT calculations applying the projector augmented plane wave method ${ }^{16}$ as implemented in Vienna $a b$ initio simulation package (VASP) ${ }^{17}$ together with the generalized gradient corrected PBE functional according to Perdew et al. ${ }^{18}$ Here a $8 \times 8 \mathrm{Ni}(100)$

(a)
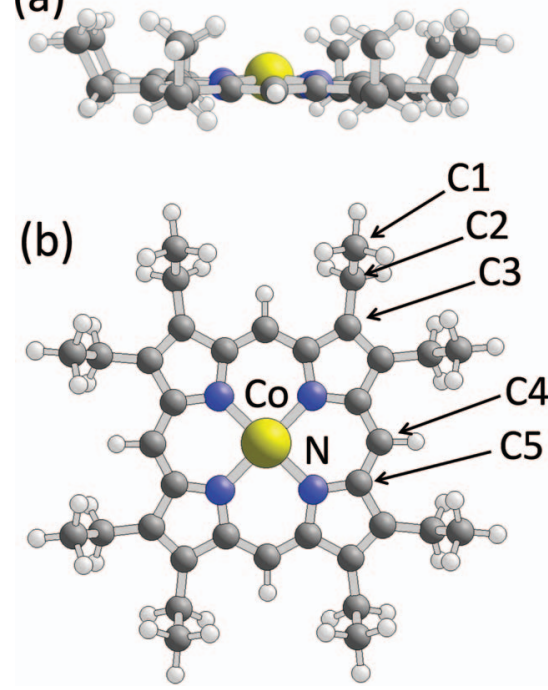

FIG. 1. Geometric structure of the free Co-OEP molecule for (a) a side view and (b) a top view. Atoms are shown by balls of different radii and labeled accordingly. surface supercell, corresponding to a periodicity length of $20.4478 \AA$ is used. As a main result, the Co-OEP is found to stabilize the most favorably with its Co center above the four-fold hollow site of the $\mathrm{Ni}(100)$ layer and its $\mathrm{N}$ atoms getting closest to $\mathrm{Ni}$ surface atoms. The $\mathrm{N}$ centers inside the adsorbed Co-OEP form a square with a N-N distance of $2.93 \AA$ which is somewhat larger than the nearest neighbor Ni-Ni distance, $2.49 \AA$, of the $\mathrm{Ni}(100)$ layer. Thus, corresponding N$\mathrm{Ni}$ bonds do not point entirely perpendicular to the surface. Further, the Co-OEP plane is curved slightly due to repulsion between the $\mathrm{Ni}$ atoms and hydrogen at the Co-OEP periphery. The shortest N-Ni distance amounts to $1.87 \AA$ which is smaller than the shortest $\mathrm{C}-\mathrm{Ni}$ distance $2.03 \AA$ at the surface. Therefore, the electronic coupling of the Co-OEP adsorbate with the $\mathrm{Ni}$ substrate may be expected to involve nitrogen atoms of the adsorbate to a larger extent than carbon atoms.

The optimized geometry of the free Co-OEP molecule as well as of the Co-OEP adsorbate at the clean Ni(100) surface from the periodic DFT model calculations serves as a basis to construct model clusters for the evaluation of theoretical core excitation spectra of free and adsorbed Co-OEP. As mentioned above, the geometry data suggest stronger electronic coupling with the $\mathrm{Ni}$ substrate for the $\mathrm{N}$ atoms than for the $\mathrm{C}$ atoms of Co-OEP. Therefore, the substrate part of the Ni/CoOEP adsorbate system is modeled by a small cluster of four $\mathrm{Ni}$ atoms $(2 \times 2$ array $)$ of the first Ni surface layer closest to and underneath the central nitrogen atoms of the adsorbate in their geometry taken from the surface optimizations, see Fig. 2(a). The resulting $\mathrm{Ni}_{4}-(\mathrm{Co}-\mathrm{OEP})$ cluster is used to calculate theoretical $\mathrm{N}$ and $\mathrm{C} 1 \mathrm{~s}$ core excitations of Co-OEP modeling the adsorbate system which will be presented below. It must be emphasized that this simulation is rather crude due to the limited size of the substrate cluster. However, qualitative trends are found to be visible already in this approximation. This is also suggested by preliminary test calculations with larger and computationally more demanding clusters including $\mathrm{Ni}_{12}$ and $\mathrm{Ni}_{16}$ substrate parts (truncated and complete planar $4 \times 4$ arrays).

In addition to the $\mathrm{Ni}_{4}-(\mathrm{Co}-\mathrm{OEP})$ cluster simulating the Co-OEP adsorbate at the clean $\mathrm{Ni}(100)$ surface, the adsorption of Co-OEP at the Ni surface covered with $\frac{1}{2}$ ML oxygen (a)

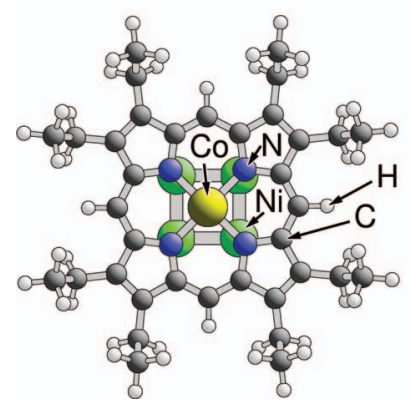

(b)

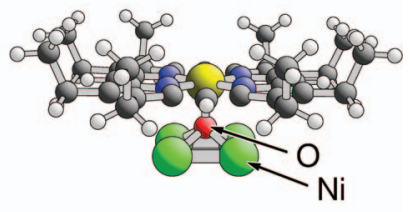

FIG. 2. Geometric structure of model clusters representing Co-OEP adsorbed at the clean and oxygen-covered Ni(100) surface, see text. (a) Top view of the $\mathrm{Ni}_{4}$-(Co-OEP) cluster; (b) side view of the $\mathrm{Ni}_{4} \mathrm{O}-(\mathrm{Co}-\mathrm{OEP})$ cluster. Atoms are shown by balls of different radii and labeled accordingly. 
(in $\mathrm{c}(2 \times 2)$ geometry) is modeled by a $\mathrm{Ni}_{4} \mathrm{O}-(\mathrm{Co}-\mathrm{OEP})$ cluster. This includes, apart from the representation of the Ni substrate by a $\mathrm{Ni}_{4}$ cluster, one oxygen atom between $\mathrm{Ni}_{4}$ and CoOEP located in the substrate hollow site as shown in Fig. 2(b). The oxygen is directly below the cobalt atom of the Co-OEP at a distance $\mathrm{d}(\mathrm{O}-\mathrm{Co})=1.74 \AA$ as evaluated by optimizing the geometry of Co-OEP with one oxygen atom binding to the central Co atom.

\section{B. NEXAFS spectrum calculations}

The local clusters obtained from the geometry optimizations discussed above are used to evaluate electronic ground as well as nitrogen and carbon 1s core excited states within DFT. In the calculations the gradient corrected revised Perdew-Burke-Ernzerhof (RPBE) exchange-correlation functional ${ }^{18,19}$ is employed and all calculations are performed with the cluster code StoBe. ${ }^{20}$

The computation of theoretical $\mathrm{C}$ and $\mathrm{N}$ 1s $\mathrm{x}$-ray absorption spectra of the Co-OEP molecule in the different clusters considers core to unoccupied orbital excitations resulting from dipole transitions. Thus, polarization-resolved spectral intensities $\mathrm{I}(\mathrm{E}, \mathrm{e})$ are determined by corresponding dipole transition matrix elements, vectors $\underline{m}=\left(m_{x}, m_{y}, m_{z}\right)$, together with angle-dependent parameters of the incoming radiation, characterized by the polarization vector $\underline{\mathrm{e}}=\left(\mathrm{e}_{\mathrm{x}}, \mathrm{e}_{\mathrm{y}}\right.$, $\mathrm{e}_{\mathrm{z}}$ ), as

$$
\mathrm{I}(\mathrm{E}, \underline{\mathrm{e}})=\kappa \cdot \mathrm{E} \cdot(\underline{\mathrm{m}} \underline{\mathrm{e}})^{2}, \quad \underline{\mathrm{m}}=\left\langle\varphi_{f}|\mathrm{q} \cdot \underline{\mathrm{r}}| \varphi_{\text {core }}\right\rangle .
$$

Here $\kappa$ is a global scaling factor, $\mathrm{E}$ denotes the transition energy, and transition dipole vectors $\underline{m}$ involve the initial core orbital $\varphi_{\text {core }}$ and excited final state orbitals $\varphi_{f}$. In addition, averaging (1) over all polarization directions in the case of disorder yields the polarization-averaged intensity as

$\mathrm{I}(\mathrm{E})=\int \mathrm{I}(\mathrm{E}, \underline{\mathrm{e}}) \mathrm{d} \Omega=2 \pi / 3 \cdot \kappa \cdot \mathrm{E} \cdot\left(\mathrm{m}_{\mathrm{x}}^{2}+\mathrm{m}_{\mathrm{y}}^{2}+\mathrm{m}_{\mathrm{z}}^{2}\right)$.

Both polarization-averaged and polarization-resolved intensities will be considered in the analysis. The evaluation of all core excited final states with corresponding transition energies $\mathrm{E}$ and matrix elements $\mathrm{m}$ is achieved within the transition potential approach ${ }^{21}$ in combination with a double basis set technique. ${ }^{22}$ This approximation involves a half-occupied $1 \mathrm{~s}$ core orbital at the excitation site, nitrogen or carbon in Co-OEP, thereby accounting for partial electronic relaxation due to the presence of the excited electron. ${ }^{23}$ The transition energies and corresponding dipole transition matrix elements in (1), (2) are convoluted using Gaussian broadening of varying width to simulate instrumental, vibrational, and life-time broadening. For both carbon (nitrogen) 1s excitations a full width at half maximum (FWHM) value of $0.5 \mathrm{eV}(1.2 \mathrm{eV})$ is applied below the ionization threshold while the broadening is increased linearly to $2 \mathrm{eV}(4 \mathrm{eV})$ up to $20 \mathrm{eV}$ above threshold and kept fixed at this value for higher energies.

In the transition potential approach the electronic core hole relaxation of the excited final state is not fully accounted for. This incomplete relaxation can be corrected in an approximate way by shifting all excitation energies by the difference of the ionization potential computed with the transition poten- tial method and the corresponding value from $\Delta$ Kohn-Sham $(\triangle \mathrm{SCF})$ calculations. This results in a global downward shift of about $2 \mathrm{eV}$, depending on the excitation site. Further, relativistic corrections are included by applying an additional upward shift of the computed spectra by $0.08 \mathrm{eV}$ for $\mathrm{C} 1 \mathrm{~s}$ excitation and $0.18 \mathrm{eV}$ for $\mathrm{N} 1 \mathrm{~s}$ excitation. ${ }^{24}$ Successful applications of the present approach to gas phase, adsorbed molecules, and surfaces can be found in Refs. 25-34. For further details of the method consult Refs. 21-26.

\section{EXPERIMENTAL DETAILS}

X-ray absorption (NEXAFS) measurements were performed for the different adsorbate systems with the substrate consisting of a $\mathrm{Cu}(100)$ single crystal covered by a thin epitaxial Ni film with or without adsorbed oxygen. The sample preparation was carried out under ultrahigh vacuum conditions ( $\mathrm{p}=2 \times 10^{-10}$ mbar). A $\mathrm{Cu}(100)$ single crystal was cleaned by cycles of $\mathrm{Ar}^{+}$sputtering at $1.0 \mathrm{keV}$ and annealing to $900 \mathrm{~K}$ where the surface quality was checked by lowenergy electron diffraction. Epitaxial Ni films were produced by electron-beam evaporation either on the clean or on a preoxidized $\mathrm{Cu}(100)$ single crystal at room temperature. The oxidized $\mathrm{Cu}(100)$ surface was prepared following Ref. 35. $\mathrm{Ni}$ growth on the $\mathrm{Cu}$ surface covered by 0.5 monolayers of atomic oxygen occurs in a surfactant-assisted mode ${ }^{35,36}$ with the oxygen atoms in $\mathrm{c}(2 \times 2)$ positions.

Co(II)-2,3,7,8,12,13,17,18-octaethylporphyrin molecules (Co-OEP), purchased from Sigma-Aldrich, were evaporated by sublimating molecular powder from a crucible at about $485 \mathrm{~K}$ onto the sample held at room temperature. The thickness of the Ni film and the coverage with porphyrin molecules were determined by intensity oscillations of medium-energy electron diffraction and by a quartz microbalance, respectively, and were cross-checked by the signal-to-background ratio edge jump at the respective $\mathrm{x}$-ray absorption edges. A coverage of Co-OEP in the submonolayer regime ensures direct contact between the molecules and the substrate. We deposited 0.7 monolayers (ML) of Co-OEP on oxygen-covered $\mathrm{Ni}$ films, while on the bare $\mathrm{Ni}$ substrates samples with a molecular coverage of 0.8 and $0.6 \mathrm{ML}$ were prepared for measurements at the $\mathrm{N} \mathrm{K}$ and the $\mathrm{C} \mathrm{K}$ edges, respectively. A full ML corresponds to a coverage of about 0.8 molecules $/ \mathrm{nm}^{2}$. Bulk porphyrin reference samples were prepared by pressing molecular powder onto a thin indium foil.

NEXAFS measurements were performed using linearly p-polarized x-rays of the helical undulator beam line UE56/2PGM1 (bulk Co-OEP and Co-OEP on Ni films) and the bending magnet beam line PM3 (Co-OEP on O-Ni) of BESSY II in Berlin, with a degree of polarization larger than $95 \%$. Spectra were acquired in total-electron-yield mode by recording the sample drain current as a function of photon energy. They were normalized to the total electron yield of a freshly evaporated gold grid upstream to the experiment and subsequently divided by the corresponding spectra of a bare and oxygen-covered Ni film, respectively, without adsorption of Co-OEP, which were also normalized to the electron yield of the gold grid. Spectra were taken between normal and grazing incidence, defined by angles of $0^{\circ}, 54^{\circ}$, and $70^{\circ}$, respectively, 


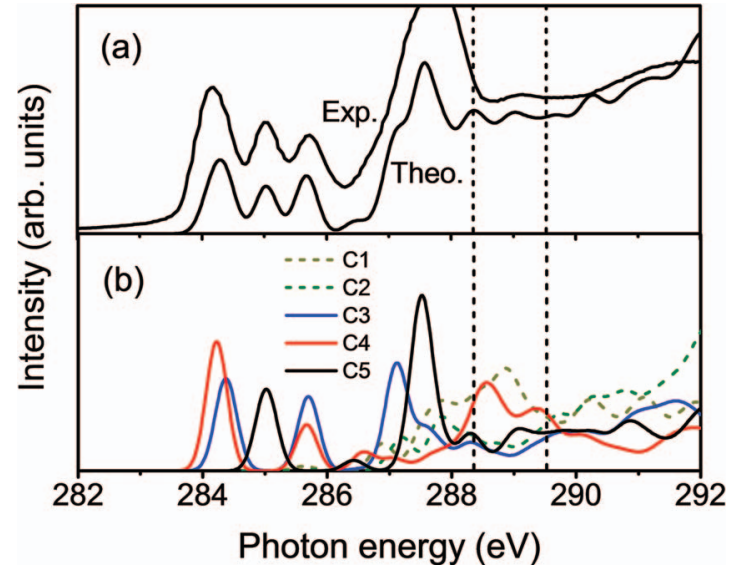

FIG. 3. (a) Theoretical C 1s core excitation spectrum of a free Co-OEP molecule (total spectrum, "Theo.") compared with experimental C K-edge NEXAFS data ("Exp.") for crystalline Co-OEP powder. (b) Decomposition of the total theoretical spectrum into contributions from the five nonequivalent carbon species, $\mathrm{C} 1$ to $\mathrm{C} 5$, in free Co-OEP, see Fig. 1(b). The two dashed vertical lines indicate the energy range of the computed ionization potentials for $\mathrm{C} 1$ to $\mathrm{C} 5$.

between the direction of the x-ray beam and the surface normal. The photon energy resolution was set to $100 \mathrm{meV}$ at the $\mathrm{C}$ K-edge, and to $150 \mathrm{meV}$ at the $\mathrm{N} \mathrm{K}$-edge. Calibration of the photon energy was carried out by means of absorption measurements of gaseous $\mathrm{N}_{2}$, setting the position of the first $\mathrm{N} \pi *$ resonance to $400.88 \mathrm{eV}^{37}$ Low photon flux densities at the sample of about $10^{13} \mathrm{~s}^{-1} \mathrm{~cm}^{-2}$ were used to prevent radiation damage. This is confirmed by comparisons of spectra taken immediately after sample preparation and at later times.

In the following we apply the experimental energy scale with the above calibration in all graphs where experimental spectra are presented (Figs. 3, 5(b)-5(d), 6(a), 7(b)-7(d), and 9(a)). This allows direct comparison with future experiments. If experimental spectra are compared with those from theory (Figs. 3, 5(b)-5(d), and 7(b)-7(d)), we apply an additional global shift $(0.3 \mathrm{eV}$ to lower energies for $\mathrm{C} 1 \mathrm{~s}, 0.3 \mathrm{eV}$ to higher energies for $\mathrm{N} 1 \mathrm{~s}$ ) to all theoretical spectra in order to facilitate the visual comparison of peak separations. In all graphs with theoretical spectra only (Figs. 4, 5(a), 6(b), 7(a), 8 , and 9(b)) we use the theoretical energy scale to allow immediate comparison with subsequent calculations. However, we point out that for all qualitative comparisons of the spectra and their interpretation these shifts can be safely ignored.

\section{RESULTS AND DISCUSSION}

\section{A. Carbon K-edge NEXAFS spectra for Co-OEP}

Figure 3(a) shows the calculated carbon 1s core excitation spectrum for a free Co-OEP molecule ("Theo.") and compares with experimental C K-edge NEXAFS data ("Exp.") obtained at $300 \mathrm{~K}$ for crystalline Co-OEP powder. The crystalline Co-OEP powder yields a rather broad peak of large intensity near $287.5 \mathrm{eV}$ (included only in parts in Fig. 3(a)) and a triple-peak structure in the energy range between $284 \mathrm{eV}$ and $286 \mathrm{eV}$. The latter three peaks at $284.3 \mathrm{eV}$, $285.0 \mathrm{eV}$, and $285.7 \mathrm{eV}$ are close in width, $0.5 \mathrm{eV}$,
$0.4 \mathrm{eV}$, and $0.45 \mathrm{eV}$ FWHM, respectively. The theoretical carbon 1s core excitation spectrum for a free Co-OEP molecule reveals also a triple-peak structure in the energy range between $284.0 \mathrm{eV}$ and $286.0 \mathrm{eV}$ and a large broad peak at $287.7 \mathrm{eV}$, near the ionization threshold. While the peak separations of the triple-peak structure are slightly smaller than in experiment, the comparison between theory and experiment suggests good agreement up to the ionization threshold. (The energy range of the computed ionization potentials for the different carbon species in Co-OEP is shown by dashed lines in Fig. 3). The small differences can be explained by the fact that the crystalline powder sample used in the experiment allows for weak electronic coupling between adjacent CoOEP molecules which is not described by the calculations for a gas phase molecule.

Figure 3(b) shows a decomposition of the total theoretical spectrum into contributions from the five non-equivalent carbon species in free Co-OEP, denoted $\mathrm{C} 1$ to $\mathrm{C} 5$, see Fig. 1(b). Obviously, contributions from the $\mathrm{C} 1$ and $\mathrm{C} 2$ species, located at the ethyl periphery of the molecule, appear only above $287 \mathrm{eV}$, i.e., energetically well outside the triple-peak region. On the other hand, the triple-peak structure in the energy range between 284 and $286 \mathrm{eV}$ is identified as being almost exclusively due to core excitations of carbon species $\mathrm{C} 3, \mathrm{C} 4$, and $\mathrm{C} 5$ residing in the inner porphyrin part of Co-OEP, see Fig. 1(b), where the peaks discriminate clearly between the species. The two outer peaks, at $284.3 \mathrm{eV}$ and $285.7 \mathrm{eV}$, originate from excitations at both $\mathrm{C} 3$ and $\mathrm{C} 4$ of about the same intensity. In contrast, the central peak at $285.1 \mathrm{eV}$ is determined by excitations at the C5 species which is next to nitrogen in the molecule. Finally, the large peak near $287 \mathrm{eV}$ in the total spectrum, i.e., near the ionization threshold, contains contributions from core excitations at all carbon sites $\mathrm{C} 1$ to $\mathrm{C} 5$ where those at $\mathrm{C} 3$ and $\mathrm{C} 5$ dominate.

More detailed information about the characteristics of the $\mathrm{C}$ core excitations can be obtained by analyses of corresponding final state orbitals. As examples, Fig. 4 shows the partial spectra obtained for core excitations at C3, C4, and C5, see Fig. 1(b). The spectra also include discrete excitation energies given by vertical lines of lengths characterizing corresponding excitation probabilities. In addition, iso-surface plots of final state orbitals of selected excitations (1), (2) are shown above each spectrum. The analysis shows first that all final state orbitals involved in the core excitations of the triple-peak structure between $284 \mathrm{eV}$ and $287 \mathrm{eV}$ and the large intensity peak below ionization threshold are described as $\pi *$ type with respect to the porphyrin plane. Here bonding/antibonding C $2 p$ mixtures (with smaller $\mathrm{N} 2 \mathrm{p}$ contributions) dominate where the amount of antibonding character determines the energetic order of the excitations in a complex way. This is obvious from the orbital plots of Figs. 4(a)-4(c) where the C 2p character at the excitation center is always combined with antibonding $2 \mathrm{p}$ functions at nearby carbon centers. Further, the final state orbitals of Figs. 4(a)-4(c) include only little $2 p$ contributions from nitrogen near the molecule center. Thus, corresponding excitations are not expected to be influenced strongly by bond formation of the nitrogen with a metal substrate when the Co-OEP molecule adsorbs at the surface. This will be discussed below. 
(a)

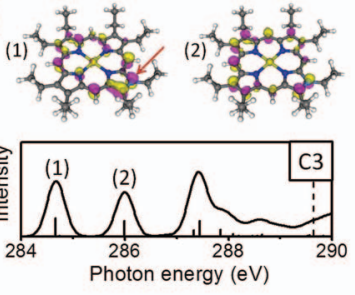

(b)
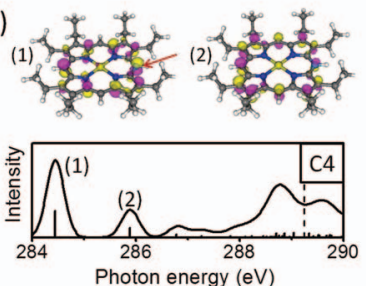

(c)
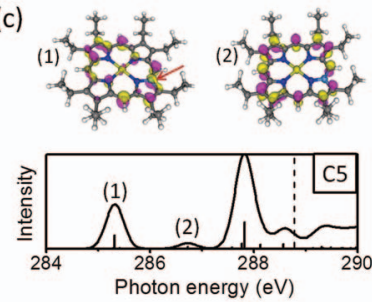

FIG. 4. Theoretical C 1s core excitation spectra of different carbon species in free Co-OEP, for (a) C3, (b) C4, and (c) C5, see Fig. 1(b). The spectra, taken from Fig. 3(b), include discrete excitation energies between $284 \mathrm{eV}$ and $287 \mathrm{eV}$ given by vertical lines of lengths characterizing corresponding excitation probabilities. The dashed vertical lines indicate the computed ionization potentials for the carbon species. The iso-surface plots above each spectrum illustrate representative final state orbitals (1), (2) of selected peaks labeled accordingly. The arrows in the plots point at corresponding carbon excitation centers.

Interestingly, for excitations at $\mathrm{C} 3, \mathrm{C} 4$, and $\mathrm{C} 5$ the two lowest peaks (1), (2) are always separated energetically by $1.5 \mathrm{eV}$, where those for $\mathrm{C} 3$ and $\mathrm{C} 4$ are energetically very similar, while the two C5-derived peaks are shifted to higher energy by $0.8 \mathrm{eV}$ with the second peak yielding only little intensity and explaining the triple-peak structure. This may suggest additional local charging at the $\mathrm{C} 5$ carbon atom (closest to nitrogen in the molecule) which is confirmed qualitatively by population analyses. However, a quantitative account of the effect remains difficult.

Figure 5(a) compares the theoretical polarizationaveraged $\mathrm{C} 1 \mathrm{~s}$ core excitation spectrum of the free Co-OEP

(a)

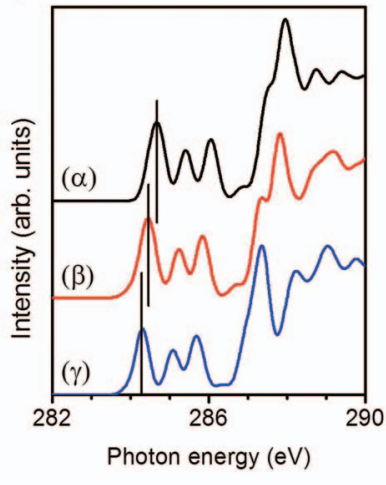

(b)

(c)

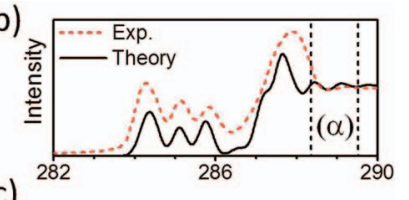

(d)

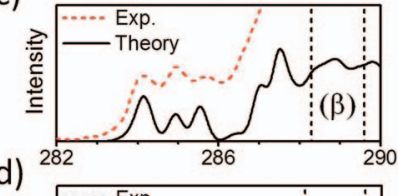

FIG. 5. (a) Theoretical polarization-averaged $\mathrm{C} 1 \mathrm{~s}$ core excitation spectra for $(\alpha)$ the free Co-OEP molecule, $(\beta)$ the $\mathrm{Ni}_{4} \mathrm{O}-(\mathrm{Co}-\mathrm{OEP})$ cluster, and $(\gamma)$ the $\mathrm{Ni}_{4}-(\mathrm{Co}-\mathrm{OEP})$ cluster, see text and Figs. 1 and 2 . The vertical lines indicate positions of the energetically lowest peak in each spectrum. (b)-(d) Comparison of the theoretical spectra $(\alpha),(\beta),(\gamma)$ with corresponding experimental polarization-averaged C K-edge NEXAFS data, see text. The vertical dotted lines indicate positions of the theoretical ionization threshold in each spectrum.

molecule with corresponding spectra for the $\mathrm{Ni}_{4} \mathrm{O}-(\mathrm{Co}-\mathrm{OEP})$ and $\mathrm{Ni}_{4}-(\mathrm{Co}-\mathrm{OEP})$ clusters used to simulate the electronic coupling of Co-OEP with a $\mathrm{Ni}(100)$ surface with and without oxygen coverage. The total spectra result from stoichiometrically weighted superpositions of partial spectra of core excitations at $\mathrm{C} 1$ to $\mathrm{C} 5$, see Figs. 1 and 2. The comparison reveals an interesting behavior of the spectra in their dependence on the electronic coupling of the Co-OEP molecule with the metal clusters which is believed to be relevant also for the extended adsorbate system. The total spectra of the three clusters are quite similar exhibiting a triple-peak structure at lower energy where the separation between the peaks is almost identical. The only difference is a small global shift of the peaks by $0.2 \mathrm{eV}$ to lower energy in going from the free molecule to that coupling with the $\mathrm{Ni}_{4} \mathrm{O}$ subunit and a shift by $0.4 \mathrm{eV}$ when Co-OEP interacts directly with the $\mathrm{Ni}_{4}$ subunit. Here the oxygen of the $\mathrm{Ni}_{4} \mathrm{O}$ subunit can be thought of as a spacer atom weakening the electronic coupling of the Co-OEP molecule with the $\mathrm{Ni}_{4}$ cluster, see Fig. 2(b), which could explain the different size of the spectral shifts. Thus, the overall weak dependence of the spectra on the electronic substrate coupling in the present models suggests that corresponding carbon $\mathrm{K}$ edge NEXAFS spectra of adsorbed Co-OEP are quite close to those of the unperturbed free molecule.

Figures 5(b)-5(d) compare calculated carbon 1s core excitation spectra for free $\mathrm{Co}-\mathrm{OEP}, \mathrm{Ni}_{4} \mathrm{O}-(\mathrm{Co}-\mathrm{OEP})$, and $\mathrm{Ni}_{4}$-(Co-OEP) with corresponding experimental C K-edge NEXAFS data for (b) crystalline Co-OEP powder, (c) CoOEP adsorbed on oxygen-covered $\mathrm{Ni}(100)$ acquired at $140 \mathrm{~K}$, and (d) Co-OEP on clean $\mathrm{Ni}(100)$ acquired at $300 \mathrm{~K}$, where the theoretical spectra are taken from Fig. 5(a). Figure 5(b) refers to an experimental spectrum of the disordered Co-OEP bulk sample (of Fig. 3) while in Figs. 5(c) and 5(d) the experimental spectra are represented by polarization-resolved spectra for magic angle photon incidence $\left(\theta=54^{\circ}\right)$. Here the theoretical spectra are shifted by $0.3 \mathrm{eV}$ to lower energies to facilitate a visual comparison of peak separations. Obviously, the experimental results confirm the theoretical triple-peak structure in the energy range between $283.5 \mathrm{eV}$ and $286.0 \mathrm{eV}$ rather nicely.

In addition, polarization-resolved theoretical spectra can be compared with results from corresponding experimental NEXAFS spectra for different photon polarization directions. Figure 6(a) shows experimental polarization-resolved $\mathrm{C}$ K-edge NEXAFS spectra for Co-OEP adsorbed at the $\mathrm{c}(2 \times 2)$ oxygen covered and clean $\mathrm{Ni}(100)$ surface measured at 140 and $300 \mathrm{~K}$, respectively. Figure 6(b) exhibits corresponding theoretical $\mathrm{C}$ 1s core excitation spectra for the $\mathrm{Ni}_{4} \mathrm{O}-(\mathrm{Co}-$ $\mathrm{OEP})$ and $\mathrm{Ni}_{4}-(\mathrm{Co}-\mathrm{OEP})$ cluster. In both figures three different polar angles of incidence of the photon beam are considered, grazing $\left(\theta=70^{\circ}\right)$, magic $\left(\theta=54^{\circ}\right)$, and normal incidence $\left(\theta=0^{\circ}\right)$. For all polar angles $\theta$ the photon polarization vector lies inside the plane through the surface normal and, for simplicity, the dependence on the azimuthal angle $\varphi$ is averaged in the theoretical spectra. Obviously, there is a rather strong angle dependence of the experimental spectra for both the oxygen-covered and the clean $\mathrm{Ni}(100)$ surface, see Fig. 6(a). For grazing incidence with the polarization vector pointing almost perpendicular to the surface the spectra 
(a)

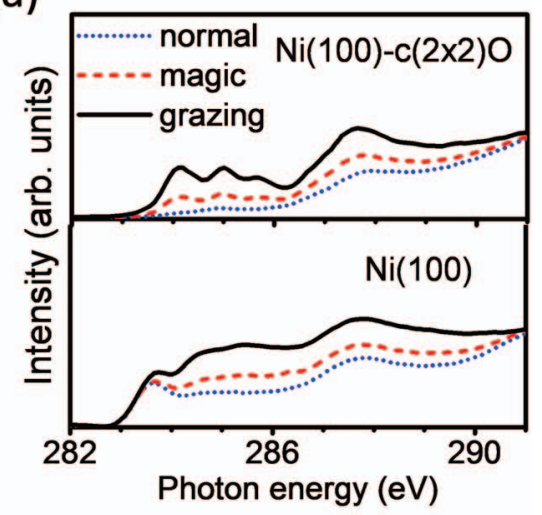

(b)

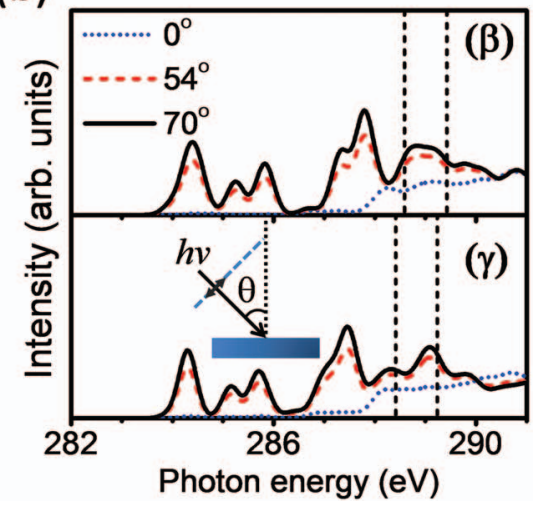

FIG. 6. (a) Experimental polarization-resolved C K-edge NEXAFS spectra for Co-OEP adsorbed on oxygen-covered $(\mathrm{c}(2 \times 2)$, top) and clean $\mathrm{Ni}(100)$ (bottom) referring to three different angles $\theta$ of photon incidence, $\theta=70^{\circ}$, $54^{\circ}, 0^{\circ}$, see text. (b) Theoretical polarization-resolved $\mathrm{C} 1 \mathrm{~s}$ core excitation spectra for the $\mathrm{Ni}_{4} \mathrm{O}-(\mathrm{Co}-\mathrm{OEP})$ cluster (top, $\left.(\beta)\right)$ and the $\mathrm{Ni}_{4}$-(Co-OEP) cluster (bottom, $(\gamma)$ ), see Figs. 2(c) and 2(d). The photon incidence angles $\theta$, sketched in the inset, are identical to those in (a). The dashed vertical lines indicate the computed ionization potentials for the carbon species.

yield triple-peak structures in the energy range between $283.5 \mathrm{eV}$ and $286.0 \mathrm{eV}$ where the peaks are much more pronounced for Co-OEP at the oxygen-covered than at the clean $\mathrm{Ni}(100)$ surface. These peaks are greatly reduced for normal photon incidence. Assuming the Co-OEP adsorbate to lie flat at the surface this angle behavior of the experimental spectra indicates strongly that the final state orbitals corresponding to excitations between $283.5 \mathrm{eV}$ and $286.0 \mathrm{eV}$ are of dominant $\pi *$ type symmetry. The effect is most obvious for the adsorbate at the oxygen-covered $\mathrm{Ni}(100)$ surface where it is weakly bound and in its electronic behavior similar to free Co-OEP.

These findings are consistent with the analysis of the corresponding final state orbitals in the calculated spectra of free Co-OEP discussed earlier. For Co-OEP at the clean Ni(100) surface the measured triple-peak structure between $283.5 \mathrm{eV}$ and $286.0 \mathrm{eV}$ is shifted to lower energy by $0.3 \mathrm{eV}$ compared with Co-OEP at the oxygen-covered $\mathrm{Ni}(100)$ surface which is consistent with the theoretical shift discussed above, see Fig. 5. The measured triple-peak structure for Co-OEP at clean $\mathrm{Ni}(100)$ is somewhat washed out. This may hint at additional intensity due to a stronger involvement of carbonderived orbitals in the electronic coupling with the $\mathrm{Ni}$ substrate. But also other effects, like the influence of tempera- ture - the spectra were taken at room temperature - cannot be excluded. Interestingly, the energetically lowest peak at $283.5 \mathrm{eV}$ in the spectra for Co-OEP at clean $\mathrm{Ni}(100)$ seems to remain strong for all polarization angles which is not yet fully understood. It may suggest that, apart from $\pi *$ type, also $\sigma^{*}$ type orbitals of Co-OEP with carbon character hybridizing with $\mathrm{Ni}$ contributions appear in the excitation spectrum. These orbitals are not fully accounted for by the small cluster models used in the calculations of the theoretical spectra. Alternatively, slight buckling of the Co-OEP adsorbate may influence the spectra since the corresponding peaks derive from carbon atoms being part of the porphinato core.

The theoretical C K-edge NEXAFS spectra for adsorbed Co-OEP, given in Fig. 6(b), yield a triple-peak structure in the energy range between $284.0 \mathrm{eV}$ and $286.0 \mathrm{eV}$ confirming the experimental spectra for bulk and adsorbed Co-OEP, see Figs. 3(a) and 6(a). Further, the dependence of the theoretical peak heights on the photon polarization direction substantiates that the origin of the peaks is due to excitations of carbon core electrons to empty orbitals of $\pi *$ type symmetry discussed earlier and compatible with the experimental findings. However, for adsorption at both the oxygen-covered and clean $\mathrm{Ni}(100)$ surface the spectral intensity between $284.0 \mathrm{eV}$ and $286.0 \mathrm{eV}$ vanishes at normal photon incidence, $\theta=0^{\circ}$. This is in contrast to the experimental spectra of Fig. 6(a) where at $\theta$ $=0^{\circ}$ there is still appreciable intensity for Co-OEP adsorbed at both substrates. This may indicate that some molecules are adsorbed at step edges revealing a different electronic structure in the experiment which needs to be investigated further.

\section{B. Nitrogen K-edge NEXAFS spectra for Co-OEP}

Figure 7(a) compares calculated polarization-averaged nitrogen 1 s core excitation spectra for $(\alpha)$ the free Co-OEP molecule, $(\beta)$ the $\mathrm{Ni}_{4} \mathrm{O}-(\mathrm{Co}-\mathrm{OEP})$ cluster, and $(\gamma)$ the $\mathrm{Ni}_{4}$ (Co-OEP) cluster, see Figs. 1 and 2. The theoretical spectra refer to one of the four nitrogen atoms in Co-OEP which
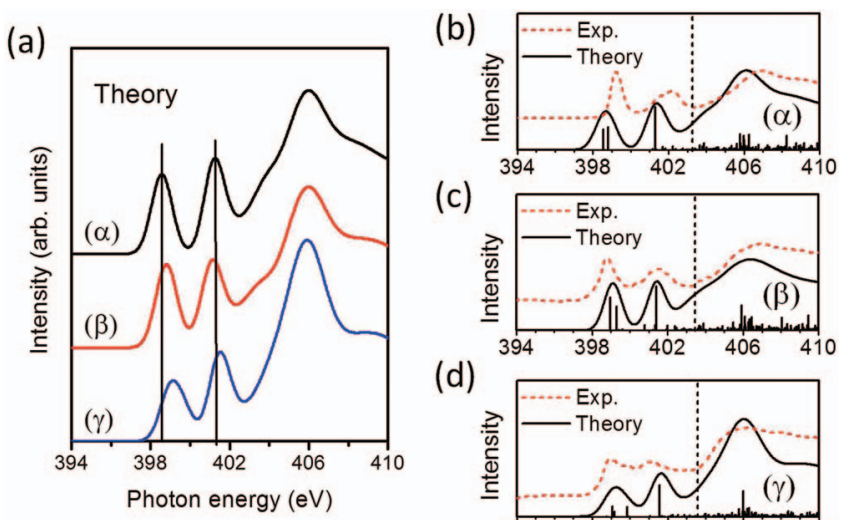

(c)

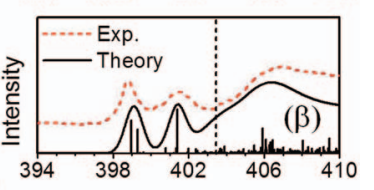

(d)

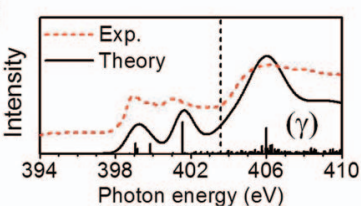

FIG. 7. (a) Theoretical $\mathrm{N} 1 \mathrm{~s}$ core excitation spectra for $(\alpha)$ the free Co-OEP molecule, $(\beta)$ the $\mathrm{Ni}_{4} \mathrm{O}-(\mathrm{Co}-\mathrm{OEP})$ cluster, and $(\gamma)$ the $\mathrm{Ni}_{4}-(\mathrm{Co}-\mathrm{OEP})$ cluster, see text and Figs. 1 and 2. The vertical lines indicate positions of the two peaks in the free Co-OEP spectrum. (b)-(d) Comparison of the theoretical spectra $(\alpha),(\beta),(\gamma)$ with corresponding experimental polarization-averaged $\mathrm{N}$ K-edge NEXAFS data, see text. The vertical dotted lines indicate positions of the theoretical ionization threshold in each spectrum. 
are equivalent due to symmetry. Clearly, all spectra exhibit a double-peak structure in the energy range between $398.0 \mathrm{eV}$ and $402.0 \mathrm{eV}$ with peaks that are similar in width and only slightly different in height. The separation between the two peaks, amounting to $2.66 \mathrm{eV}$ for free Co-OEP, is reduced somewhat to $2.40 \mathrm{eV}$ in $\mathrm{Ni}_{4} \mathrm{O}-(\mathrm{Co}-\mathrm{OEP})$ and to $2.36 \mathrm{eV}$ for $\mathrm{Ni}_{4}$-(Co-OEP) where the molecule comes closest to the metal surface. Further, the presence of the metal part in the clusters leads to additional intensity near $400.0 \mathrm{eV}$ between the two peaks which is not found for free Co-OEP. These results are understood as substrate-induced effects due to hybridization of nitrogen-type orbitals with the metal as will be discussed below.

Figures 7(b)-7(d) compare calculated nitrogen 1s core excitation spectra for free $\mathrm{Co}-\mathrm{OEP}, \mathrm{Ni}_{4} \mathrm{O}-(\mathrm{Co}-\mathrm{OEP})$, and $\mathrm{Ni}_{4}-(\mathrm{Co}-\mathrm{OEP})$ with corresponding experimental $\mathrm{N}$ K-edge NEXAFS data for (b) crystalline Co-OEP powder, (c) CoOEP adsorbed on oxygen-covered $\mathrm{Ni}(100)$, and (d) Co-OEP on clean $\mathrm{Ni}(100)$ acquired at $300 \mathrm{~K}$ where the theoretical spectra are taken from Fig. 7(a). Further, the theoretical spectra are shifted by $0.3 \mathrm{eV}$ to higher energies to facilitate a visual comparison of peak separations. Obviously, the experimental results confirm the theoretical findings rather nicely. First, the experimental spectra yield in all cases a double-peak structure in the energy range between $398.0 \mathrm{eV}$ and $402.5 \mathrm{eV}$ with peaks similar to those predicted by theory. Second, the energetic separation between the two peaks reduces in going from crystalline Co-OEP powder $(3.07 \mathrm{eV})$ to Co-OEP adsorbed on the oxygen-covered $(2.79 \mathrm{eV})$ and on the clean $\mathrm{Ni}(100)$ surface $(2.15 \mathrm{eV})$, consistent with theory. However, the measured peak separations seem to be reduced by somewhat larger amounts compared with theory. This can be simply understood by an increase in adsorbate-substrate coupling at the extended surface in the experiment compared with the approximation of the substrate by a small metal cluster in the calculations. Third, in the experimental NEXAFS spectra for the Co-OEP adsorbate at $\mathrm{Ni}(100)$ there is increased intensity near $400.0 \mathrm{eV}$ between the two peaks, also found in theory, which suggests additional excitations due to the presence of the substrate surface.

A more detailed description of the influence of the metal substrate on the electronic coupling with the Co-OEP molecule and on corresponding $\mathrm{N}$ 1s core excitations is obtained by examining final state orbitals. As an illustration, Fig. 8 compares the theoretical $\mathrm{N} 1 \mathrm{~s}$ excitation spectra for free Co-OEP and for $\mathrm{Ni}_{4}-(\mathrm{Co}-\mathrm{OEP})$ simulating the adsorbate. The spectra, taken from Fig. 7(a), include discrete excitation energies in the double-peak region above $398 \mathrm{eV}$ given by vertical lines of lengths characterizing excitation probabilities. Further, iso-surface plots of final state orbitals of selected excitations, labeled (1), (2), $\left(2^{+}\right),(3)$, are shown above each spectrum.

For free Co-OEP, the energetically lower excitation peak at $398.4 \mathrm{eV}$ in the spectrum originates from two excitations (1), (2) separated by $0.25 \mathrm{eV}$ and of about the same intensity. Here the iso-surface plot of orbital (1) evidences an antibonding mixture of central cobalt $3 \mathrm{~d}$ with $2 \mathrm{p}$ character from the four surrounding nitrogen atoms. This yields an altogether $\sigma^{*}$ type orbital with only very small contributions from the
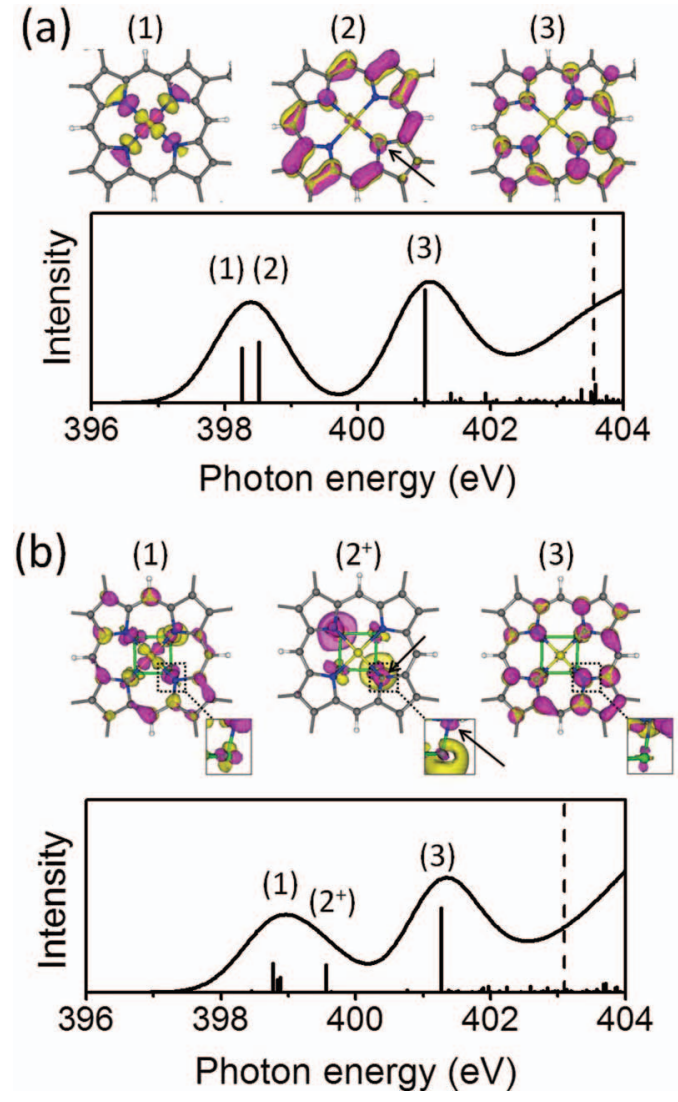

FIG. 8. Theoretical $\mathrm{N}$ 1s core excitation spectra for the (a) free Co-OEP molecule and (b) $\mathrm{Ni}_{4}$-(Co-OEP) cluster, taken from Fig. 7(a), see also text and Figs. 1 and 2. The spectra include discrete excitation energies in the doublepeak region above $398 \mathrm{eV}$ given by vertical lines of lengths characterizing excitation probabilities. The dashed vertical lines indicate the computed ionization potentials for the nitrogen species. The iso-surface plots above each spectrum illustrate representative final state orbitals of (1), (2), ( $\left.2^{+}\right),(3)$, see text. The arrows in the plots point at corresponding nitrogen excitation centers.

molecular periphery (not included in the plot). In contrast, the plot of orbital (2) reveals a $\pi^{*}$ type orbital with $N 2 p$ functions mixing with $\mathrm{C} 2 \mathrm{p}$, both bonding and antibonding, and no Co contributions. The energetically higher excitation peak at $401.2 \mathrm{eV}$ is dominated by only one excitation (3) where the final state orbital plot shows a $\pi^{*}$ type orbital with $\mathrm{N} 2 \mathrm{p}$ functions combining with $\mathrm{C} 2 \mathrm{p}$ in an antibonding fashion.

Figure 8(b) shows the calculated nitrogen 1 s core excitation spectrum for the $\mathrm{Ni}_{4}-(\mathrm{Co}-\mathrm{OEP})$ cluster together with discrete excitation energies in the double-peak region and isosurface plots of representative final state orbitals. The insets show enlarged side views of the nitrogen excitation region with the Ni atom underneath. Here the energetically lower excitation peak at $399.0 \mathrm{eV}$ is derived from three excitations which are very close in energy and located at the peak center where only orbital (1), yielding largest intensity, is shown above the spectrum. This orbital represents an antibonding mixture of central cobalt $3 \mathrm{~d}$ and nitrogen $2 \mathrm{p}$ character and is very similar in shape to the $\sigma^{*}$ type orbital (1) of free CoOEP, see Fig. 8(a). In addition, orbital (1) contains contributions from the four nickel atoms underneath whose $3 \mathrm{~d}$ orbitals hybridize with $2 p$ functions of their nitrogen neighbors in an antibonding fashion. This can explain the $0.7 \mathrm{eV}$ shift of 
the corresponding excitation energies between free Co-OEP and $\mathrm{Ni}_{4}-(\mathrm{Co}-\mathrm{OEP})$ as an adsorption-induced hybridization effect. The final state orbitals of the other two excitations near $399.0 \mathrm{eV}$ (not shown in the figure) are described as $\pi^{*}$ type with $\mathrm{N}$ and $\mathrm{C} 2 \mathrm{p}$ mixing and only very small $\mathrm{Ni} 3 \mathrm{~d}$ contributions. There is an additional excitation at $399.6 \mathrm{eV}$, denoted $\left(2^{+}\right)$which does not appear in the spectrum of free Co-OEP. The corresponding final state orbital $\left(2^{+}\right)$, shown at the top of Fig. 8(b), is characterized by large $\mathrm{Ni} 3 \mathrm{~d}$ and $4 \mathrm{~s}$ contributions with some antibonding N 2p admixture, as indicated in the inset of the orbital plot. Thus, it can be considered the hybridization partner of orbital (1) such that orbitals (1) and $\left(2^{+}\right)$ illustrate the electronic coupling between the $\mathrm{Ni}_{4}$ unit and the Co-OEP molecule in the core excited final states of the cluster simulating the adsorbate case. Corresponding occupied orbitals, described as bonding Ni $3 \mathrm{~d}$ and $\mathrm{N} 2 \mathrm{p}$ mixtures and determining the electronic $\mathrm{Ni}_{4}$ - (Co-OEP) coupling already in the cluster ground state, could be identified by detailed orbital analyses. However, they will not appear in the core excitation spectra.

As a result of the additional excitation $\left(2^{+}\right)$, the energetically lower excitation peak in Fig. 8(b) is broader than that obtained for free Co-OEP adding intensity to the energy region between the two peaks near $400 \mathrm{eV}$. This is consistent with the findings in the experimental spectrum for Co-OEP adsorbed at clean $\mathrm{Ni}(100)$, see Fig. 7(d). The energetically higher excitation peak at $401.3 \mathrm{eV}$ in Fig. 8(b) is dominated by one excitation where the corresponding final state orbital (3) is $\pi^{*}$ type with antibonding combinations of $N 2 p$ and $C$ $2 p$ functions and only minute Ni $3 \mathrm{~d}$ contributions. This orbital is very close in shape to the $\pi^{*}$ type orbital (3) of free CoOEP, see Fig. 8(a), which could also explain the rather small $0.2 \mathrm{eV}$ shift of the corresponding excitation energies between free Co-OEP and $\mathrm{Ni}_{4}-(\mathrm{Co}-\mathrm{OEP})$.

Additional information about binding properties and the geometry of the free and adsorbed Co-OEP molecule can be gained from polarization-resolved NEXAFS measurement in combination with theoretical studies. Figure 9 compares experimental polarization-resolved $\mathrm{N}$ K-edge NEXAFS spectra for Co-OEP adsorbed at the $\mathrm{c}(2 \times 2)$ oxygen covered and clean $\mathrm{Ni}(100)$ surface, taken at $300 \mathrm{~K}$, with corresponding theoretical $\mathrm{N}$ 1s core excitation spectra for the $\mathrm{Ni}_{4} \mathrm{O}-(\mathrm{Co}-\mathrm{OEP})$ and $\mathrm{Ni}_{4}$-(Co-OEP) cluster. Here three different polar angles of incidence of the photon beam are considered, grazing $\left(\theta=70^{\circ}\right)$, magic $\left(\theta=54^{\circ}\right)$, and normal incidence $\left(\theta=0^{\circ}\right)$ as discussed earlier for the $\mathrm{C}$ K-edge NEXAFS spectra.

The experimental spectra of Fig. 9(a) show for both the oxygen-covered and the clean $\mathrm{Ni}(100)$ surface the same strong angle dependence. For normal incidence when the polarization vector points parallel to the surface the spectra yield rather little intensity in the energy region between $398.0 \mathrm{eV}$ and $402.0 \mathrm{eV}$. In contrast, for grazing incidence with the polarization vector pointing almost perpendicular to the surface the same energy region yields the double-peak structure which has been discussed earlier. With the Co-OEP adsorbate assumed to lie flat at the surface this angle behavior of the spectra proves that the final state orbitals corresponding to excitations between $398.0 \mathrm{eV}$ and $402.0 \mathrm{eV}$ are of dominant $\pi *$ type symmetry with $\sigma^{*}$ type orbitals being much less (a)
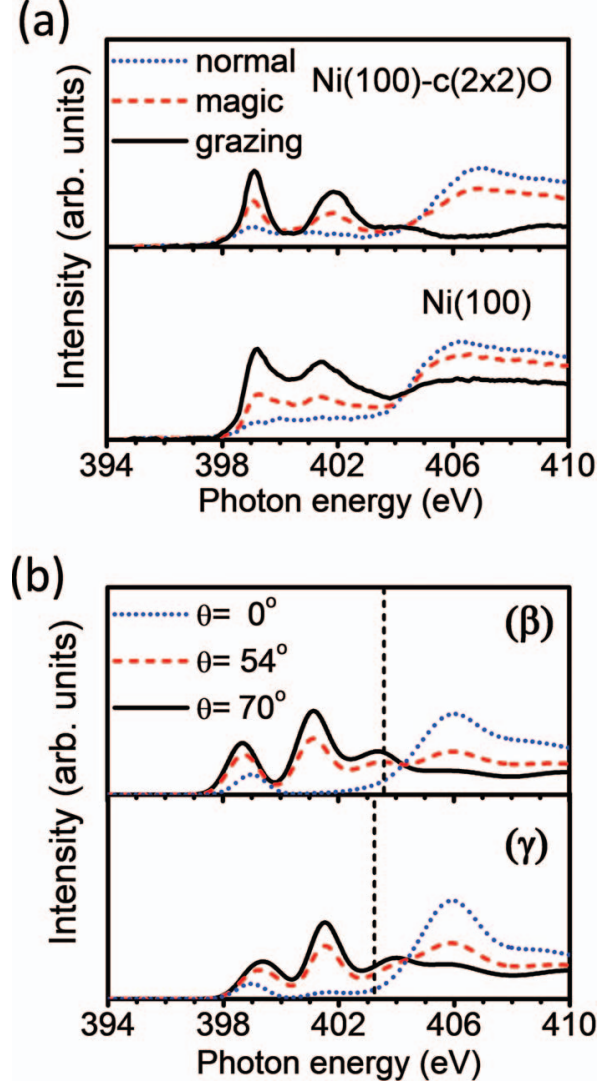

FIG. 9. (a) Experimental polarization-resolved N K-edge NEXAFS spectra for Co-OEP adsorbed on oxygen-covered (top) and clean $\mathrm{Ni}(100)$ (bottom) referring to three different angles $\theta$ of photon incidence, $\theta=70^{\circ}, 54^{\circ}, 0^{\circ}$, see text. (b) Theoretical polarization-resolved $\mathrm{N} 1 \mathrm{~s}$ core excitation spectra for the $\mathrm{Ni}_{4} \mathrm{O}-(\mathrm{Co}-\mathrm{OEP})$ cluster (top, $(\beta)$ ) and the $\mathrm{Ni}_{4}-(\mathrm{Co}-\mathrm{OEP})$ cluster (bottom, $(\gamma))$, see Figs. 2(c) and 2(d). The photon incidence angles $\theta$, sketched in the inset of Fig. 6, are identical to those in (a). The dashed vertical lines indicate the computed ionization potentials for the nitrogen species.

important. This is confirmed by the analysis of the final state orbitals in the calculated spectra discussed above with the theoretical spectra reproducing the angle dependence of the experiment, see Fig. 9(b). Here the theoretical spectra refer to different polar angles $\theta$ of the photon beam where, for simplicity, the dependence on the azimuthal angle $\varphi$ has been averaged. Interestingly, the calculations yield for both clusters and for normal photon incidence $\left(\theta=0^{\circ}\right)$ a smaller peak near $399.0 \mathrm{eV}$. This peak originates from excitations involving the $\sigma^{*}$ type final state orbitals such as (1) of Fig. 8 describing the Co-N coupling in the Co-OEP adsorbate with minor Ni $3 \mathrm{~d}$ admixture. The peak does not seem to appear or is only poorly developed in the experimental spectra. This may hint at adsorbate-substrate hybridization effects which yield additional intensity in the energy region between $398.0 \mathrm{eV}$ and $402.0 \mathrm{eV}$ and are not included in the present theoretical cluster models.

Further, the experimental spectra yield for normal incidence near $406 \mathrm{eV}$, well above the ionization threshold, a broad resonance which is damped for grazing incidence. Hence the final states of this resonance must be of $\sigma^{*}$ type symmetry. This is also found in the final state orbital analysis of the calculated spectra. The resonance orbitals exhibit dominant $\sigma$ symmetry reflecting the direct electronic $\mathrm{N}-\mathrm{Co}$ 
coupling as well as $\mathrm{C}-\mathrm{N}$ binding at the periphery of the adsorbate. This yields the same angle dependence of the spectra as found in the experiment.

\section{CONCLUSIONS}

In this study we have evaluated theoretical carbon and nitrogen 1s core excitation spectra of free Co-OEP as well as for model clusters representing the Co-OEP molecule adsorbed at local sections of the clean and oxygen-covered $\mathrm{Ni}(100)$ surface using density-functional theory methods. These results are compared first with polarization-averaged $\mathrm{C}$ and $\mathrm{N} \mathrm{K}$-edge NEXAFS spectra measured in this study for crystalline CoOEP powder and for Co-OEP adsorbed at Ni(100) surfaces with and without pre-adsorbed oxygen.

For crystalline Co-OEP powder the experimental $\mathrm{C} \mathrm{K}$ edge NEXAFS spectrum shows a triple-peak structure in the excitation energy range between $284 \mathrm{eV}$ and $286 \mathrm{eV}$, which is reproduced by the calculations on free Co-OEP. In addition, the calculations allow an assignment of the different peaks to specific carbon species in the molecule. The two outer peaks of the triple-peak structure are due to excitations at both $\mathrm{C} 3$ and $\mathrm{C} 4$ atoms, located in the inner porphyrin part of Co-OEP, while the central peak originates from excitations at $\mathrm{C} 5$ which is next to nitrogen in the molecule. The corresponding excited final state orbitals are characterized as $\pi *$ type with respect to the porphyrin plane. Excitations at $\mathrm{C} 1$ and $\mathrm{C} 2$, positioned at the ethyl periphery of the molecule, appear only at energies well above those of the triple-peak structure. The experimental N K-edge NEXAFS spectrum of crystalline Co-OEP powder shows a double-peak structure in the excitation energy range between $398.0 \mathrm{eV}$ and $402.0 \mathrm{eV}$, which is also reproduced by the calculations on free Co-OEP. Here the theoretical analysis shows that the corresponding final state orbitals characterizing the two peaks are described by $\mathrm{N} 2 \mathrm{p}$ functions mixing with $3 \mathrm{~d}$ functions of central cobalt as well as $2 \mathrm{p}$ of the neighboring carbon in an antibonding fashion. The resulting orbitals are of both $\sigma^{*}$ and $\pi^{*}$ type symmetry.

For Co-OEP submonolayers adsorbed at the $\mathrm{Ni}(100)$ surfaces, prepared as thin epitaxial films on $\mathrm{Cu}(100)$ substrate, both adsorption at the clean surface and at that with pre-adsorbed oxygen has been considered. Here the oxygen acts only as a spacer layer to increase the distance between the Co-OEP adsorbate and the $\mathrm{Ni}$ surface, thus, weakening their chemisorptive interaction. The theoretical results for $\mathrm{C}$ 1s core excitations of the Co-OEP adsorbate show that the triple-peak structure found in the NEXAFS spectrum for free Co-OEP is only weakly perturbed by adsorption, both at the clean and oxygen-covered Ni substrate. The electronic adsorbate-substrate coupling shifts the peaks rigidly by 0.2 $\mathrm{eV}$ to lower energy in going from the free molecule to that coupling with the $\mathrm{Ni}_{4} \mathrm{O}$ subunit and a shift by $0.4 \mathrm{eV}$ when Co-OEP interacts with the $\mathrm{Ni}_{4}$ subunit. The shift variation can be explained simply by the different distances between the adsorbate and the substrate part in the two models and suggests, altogether, little electronic $\mathrm{C}-\mathrm{Ni}$ coupling by orbital hybridization. This is evident from theoretical orbital analyses and is confirmed by experimental $\mathrm{C}$ K-edge NEXAFS spectra of the adsorbate systems.
The theoretical results for $\mathrm{N}$ 1s core excitations of the Co-OEP adsorbate yield double-peak structures in the spectra which are rather similar to those found for free Co-OEP and agree nicely with polarization-averaged $\mathrm{N} \mathrm{K}$-edge spectra measured for Co-OEP on clean and oxygen-covered $\mathrm{Ni}(100)$. The energy separation between the two peaks is reduced by the presence of the metal substrate, evident in both the theoretical and experimental data. Further, there is additional intensity between the two peaks found in experiment which is explained by theoretical orbital analyses as a substrateinduced effect due to hybridization of nitrogen-type orbitals with the metal.

Measured polarization-resolved $\mathrm{N}$ K-edge NEXAFS spectra together with theoretical studies for Co-OEP adsorbed at $\mathrm{Ni}(100)$ can explain further details of binding properties and the geometry of the adsorbate system. The experimental spectra exhibit a strong dependence on the polar angle $\theta$ of the photon beam with respect to the substrate surface normal. This is explained by the calculations as a consequence of symmetry of the excited final state orbitals which are dominantly $\pi^{*}$ type with only minor $\sigma^{*}$ type components resulting from $\mathrm{Ni} 3 \mathrm{~d}-\mathrm{N} 2 \mathrm{p}$ hybridization.

Altogether, the present comparison between theoretical 1s core excitation spectra and measured K-edge NEXAFS data shows that such combined theoretical and experimental studies can help to elucidate general physical and chemical behavior, which goes beyond their relevance for specific adsorbate systems.

\section{ACKNOWLEDGMENTS}

Financial support by the German Research Foundation (DFG) through the Joint Collaborative Research Center "Elementary processes in molecular switches at surfaces" (SFB 658 ) is gratefully acknowledged. M. Mesta performed preliminary calculations on core excitations in free Co-OEP molecules. We thank A. Krüger and J. Miguel for performing the NEXAFS measurements together with C.F.H. and M.B. B. Zada, W. Mahler, and T. Kachel provided technical assistance during the beam time at BESSY-II.

${ }^{1}$ S. Venkataramani, U. Jana, M. Dommaschk, F. D. Sönnichsen, F. Tuczek, and R. Herges, Science 331, 445 (2011).

${ }^{2}$ A. Scheybal, T. Ramsvik, R. Bertschinger, M. Putero, F. Nolting, and T. A. Jung, Chem. Phys. Lett. 411, 214 (2005).

${ }^{3}$ H. Wende, M. Bernien, J. Luo, C. Sorg, N. Ponpandian, J. Kurde, J. Miguel, M. Piantek, X. Xu, P. Eckhold, W. Kuch, K. Baberschke, P. M. Panchmatia, B. Sanyal, P. M. Oppeneer, and O. Eriksson, Nature Mater. 6, 516 (2007).

${ }^{4}$ M. Bernien, X. Xu, J. Miguel, M. Piantek, P. Eckhold, J. Luo, J. Kurde, W. Kuch, K. Baberschke, H. Wende, and P. Srivastava, Phys. Rev. B 76, 214406 (2007)

${ }^{5}$ D. Chylarecka, T. K. Kim, K. Tarafder, K. Müller, K. Gödel, I. Czekaj, C. Wäckerlin, M. Cinchetti, M. E. Ali, C. Piamonteze, F. Schmitt, J.-P. Wüstenberg, C. Ziegler, F. Nolting, M. Aeschlimann, P. M. Oppeneer, N. Ballav, and T. A. Jung, J. Phys. Chem. C 115, 1295 (2011).

${ }^{6}$ M. Bernien, J. Miguel, C. Weis, M. E. Ali, J. Kurde, B. Krumme, P. M. Panchmatia, B. Sanyal, M. Piantek, P. Srivastava, K. Baberschke, P. M. Oppeneer, O. Eriksson, W. Kuch, and H. Wende, Phys. Rev. Lett. 102, 047202 (2009)

${ }^{7}$ D. Chylarecka, C. Wäckerlin, T. K. Kim, K. Müller, F. Nolting, A. Kleibert, N. Ballav, and T. A. Jung, J. Phys. Chem. Lett. 1, 1408 (2010).

${ }^{8}$ C. Wäckerlin, D. Chylarecka, A. Kleibert, K. Müller, C. Iacovita, F. Nolting, T. A. Jung, and N. Ballav, Nat. Commun. 1, 61 (2010). 
${ }^{9}$ J. Miguel, C. F. Hermanns, M. Bernien, A. Krüger, and W. Kuch, J. Phys. Chem. Lett. 2, 1455 (2011).

${ }^{10}$ S. A. Krasnikov, A. B. Preobrajenski, N. N. Sergeeva, M. M. Brzhezinskaya, M. A. Nesterov, A. A. Cafolla, M. O. Senge, and A. S. Vinogradov, Chem. Phys. 332, 318 (2007).

${ }^{11} \mathrm{~S}$. Narioka, H. Ishii, Y. Ouchi, T. Yokoyama, T. Ohta, and K. Seki, J. Phys. Chem. 99, 1332 (1995).

${ }^{12}$ M. G. Betti, P. Gargiani, R. Frisenda, R. Biagi, A. Cossaro, A. Verdini, L. Floreano, and C. Mariani, J. Phys. Chem. C 114, 21638 (2010).

${ }^{13}$ M. Fanetti, A. Calzolari, P. Vilmercati, C. Castellarin-Cudia, P. Borghetti, G. Di Santo, L. Floreano, A. Verdini, A. Cossaro, I. Vobornik, E. Annese, F. Bondino, S. Fabris, and A. Goldoni, J. Phys. Chem. C 115, 11560 (2011).

${ }^{14}$ W. Auwärter, F. Klappenberger, A. Weber-Bargioni, A. Schirin, T. Strunskus, C. Wöll, Y. Pennec, A. Riemann, and J. V. Barth, J. Am. Chem. Soc. 129, 11279 (2007).

${ }^{15}$ M. Bernien, Ph.D. dissertation, Freie Universität, Berlin, 2010.

${ }^{16}$ P. E. Blöchl, Phys. Rev. B 50, 17953 (1994).

${ }^{17}$ G. Kresse and J. Furthmüller, Comput. Mater. Sci. 6, 15 (1996); Phys. Rev. B 54, 11169 (1996).

${ }^{18}$ J. P. Perdew, K. Burke, and M. Ernzerhof, Phys. Rev. Lett. 77, 3865 (1996).

${ }^{19}$ B. Hammer, L. B. Hansen, and J. K. Nørskov, Phys. Rev. B 59, 7413 (1999).

${ }^{20}$ K. Hermann and L. G. M. Pettersson, deMon developers group, StoBe software V. 3.1, 2011; see http://www.fhi-berlin.mpg.de/KHsoftware/StoBe/.

${ }^{21}$ L. Triguero, L. G. M. Pettersson, and H. Ågren, Phys. Rev. B 58, 8097 (1998).

${ }^{22}$ H. Ågren, V. Carravetta, O. Vahtras, and L. G. M. Pettersson, Chem. Phys. Lett. 222, 75 (1994)

${ }^{23}$ M. Leetmaa, M. P. Ljungberg, A. Lyubartsev, A. Nilsson, and L. G. M. Pettersson, J. Electron Spectrosc. Relat. Phenom. 177, 135 (2010).
${ }^{24}$ O. Takahashi and L. G. M. Pettersson, J. Chem. Phys. 121, 10339 (2004).

${ }^{25}$ C. Kolczewski, R. Püttner, O. Plashkevych, H. Ågren, V. Staemmler, M. Martins, G. Snell, A. S. Schlachter, M. Sant'Anna, G. Kaindl, L. G. M. Pettersson, J. Chem. Phys. 115, 6426 (2001).

${ }^{26}$ C. Kolczewski and K. Hermann, Surf. Sci. 552, 98 (2004).

${ }^{27}$ M. Cavalleri, M. Odelius, D. Nordlund, A. Nilsson, and L. G. M. Pettersson, Phys. Chem. Chem. Phys. 7, 2854 (2005).

${ }^{28}$ C. Kolczewski, R. Püttner, M. Martins, A. S. Schlachter, G. Snell, M. Sant'Anna, K. Hermann, and G. Kaindl, J. Chem. Phys. 124, 034302 (2006).

${ }^{29}$ M. Cavalleri, L. Å. Näslund, D. C. Edwards, P. Wernet, H. Ogasawara, S. Myneni, L. Öjamäe, M. Odelius, A. Nilsson, and L. G. M. Pettersson, J. Chem. Phys. 124, 194508 (2006).

${ }^{30} \mathrm{P}$. Wernet, D. Nordlund, U. Bergmann, M. Cavalleri, M. Odelius, H. Ogasawara, L. Å. Näslund, T. K. Hirsch, L. Öjamäe, P. Glatzel, L. G. M. Pettersson, and A. Nilsson, Science 304, 995 (2004).

${ }^{31}$ H. Öström, H. Ogasawara, L. Å. Näslund, L. G. M. Pettersson, and A. Nilsson, Phys. Rev. Lett. 96, 146104 (2006).

${ }^{32}$ T. Schiros, S. Haq, H. Ogasawara, O. Takahashi, H. Öström, K. Andersson, L. G. M. Pettersson, A. Hodgson, and A. Nilsson, Chem. Phys. Lett. 429, 415 (2006).

${ }^{33}$ C. Kolczewski and K. Hermann, J. Chem. Phys. 118, 7599 (2003).

${ }^{34}$ M. Cavalleri, K. Hermann, S. Guimond, Y. Romanyshyn, H. Kuhlenbeck, and H.-J. Freund, Catal. Today 124, 21 (2007).

${ }^{35}$ C. Sorg, N. Ponpandian, M. Bernien, K. Baberschke, H. Wende, and R. Q. Wu, Phys. Rev. B 73, 064409 (2006).

${ }^{36}$ R. Nünthel, T. Gleitsmann, P. Poulopoulos, A. Scherz, J. Lindner, E. Kosubek, Ch. Litwinski, Z. Li, H. Wende, K. Baberschke, S. Stolbov, and T. S. Rahman, Surf. Sci. 531, 53 (2003).

${ }^{37}$ R. N. S. Sodhi and C. E. Brion, J. Electron. Spectrosc. Relat. Phenom. 34, 363 (1984). 